\title{
EXPReS and NEXPReS
}

\section{The future of European VLBI}

\author{
Arpad Szomoru ${ }^{1}$ \\ Joint Institute for VLBI in Europe \\ P.O. Box 2, 7990 AA Dwingeloo, The Netherlands \\ E-mail: szomoruejive.nI
}

Through the EC-funded EXPReS project, which ended in September 2009, e-VLBI has developed into a fully functional operational mode of the EVN. Currently 10 to 12 24-hour esessions are scheduled per year, although target-of-opportunity (ToO) and triggered projects occur more and more frequently. This new capability of the EVN to respond to transient events is illustrated by a large number of Astronomers' Telegrams and has led to a wealth of scientific publications.

Over the past years, e-VLBI has proven to be equal and even superior to tradional disk-based VLBI, in terms of sensitivity, resolution, reliability and turn-around time. In spite of this, the majority of VLBI observations are still disk-based. This is caused by factors such as the lack of high-speed connections to a number of stations and the limitations imposed by the current correlator and networking hardware.

The EC-funded NEXPReS project, which kicked off on July 12010 , will address these issues by adding an e-VLBI component to all VLBI observations, while retaining the possibility to re-do correlations through a high-speed buffering mechanism. It will also investigate international bandwidth on demand (BoD) schemes, and prepare the EVN for 4 Gbps (and higher) operations.

In this paper I will review the development of e-VLBI through EXPReS, and present the rationale and aims of NEXPReS.

10th European VLBI Network Symposium and EVN Users Meeting: VLBI and the new generation of radio arrays

Manchester, $U K$

September 20-24, 2

$1 \quad$ Speaker




\section{Introduction}

The development of European e-VLBI started in 2002, following the roll-out of Mark5 recording and playback systems in the EVN. As these systems are (at least partly) based on standard PC hardware, sending and receiving data over the internet became an immediate possibility. An official pilot project involving several National Research and Educational Networks (NRENs), a number of EVN radio observatories and JIVE followed in 2004, and led to the first real-time observations with the EVN, albeit at very modest data rates. With the start of the EC-funded EXPReS project e-VLBI moved from a technology experiment to the fullscale development of a new scientific capability for the EVN [1], [2].

At the start of EXPReS, in March 2006, several concerns were to be addressed. Would

- we be able to connect enough telescopes fast enough?

- the bandwidth be high enough?

- e-VLBI be as reliable as non-e?

- long-haul intercontinental data transport be sustainable?

- it produce new science?

- it be cost effective?

- we be able to accommodate all types of projects?

Looking back at the project, it is safe to say that all these questions have been answered affirmatively, with the possible exception of the last two. The costs of high-speed connections and solving last-mile problems have been vastly different in different locations, and costeffectiveness depends for a large part on the support, the missions and the business models of local NRENs. Neither is it possible to claim that all types of projects currently can be accomodated in e-mode.

\section{2. e-VLBI and EXPReS}

At the start of the EXPReS project, only very few telescope sites had high-speed internet access. With most telescopes located as remote from civilization as possible, solving the socalled last-mile problem in many cases meant solving the last-tens-of-miles problem. Here the EXPReS project served as a catalyst, providing small amounts of seed money, which in many cases stimulated the active involvement and support of local and national governments. At the end of the project nearly all European EVN stations had 1Gbps connections (at least) to the correlator at JIVE through their local NRENs, the GÉANT European backbone and the Dutch SURFnet. Intercontinentally, high-speed connections had been established to Chinese and Australian stations and the Arecibo telescope in Puerto Rico through the EC-sponsored TEIN network and through the lightpaths provided by the GLIF collaboration. Even the TIGO and the Hartebeesthoek stations in Chile and South Africa managed to participate in several demonstration experiments, although at low data rates (Figure 1). 


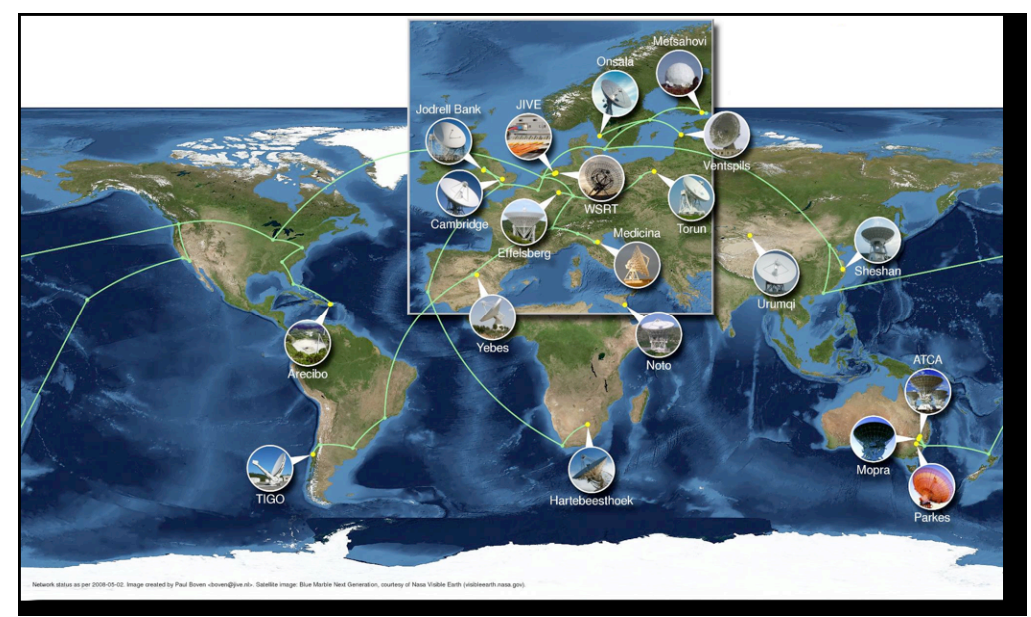

Figure 1: Map depicting all EXPReS telescopes connected to the correlator in real-time in the course of the project

Different data transport protocols and their performance were intensively studied during the early phases of the project (Figure 2). TCP, a protocol meant to be fair to all users, was found to be unusable on long-haul connections. UDP proved to be much more suitable, but necessitated an extensive re-write of the Mark 5 control code. It also can be quite hostile to other users, which means care had to be taken to make good agreements with providers. As much as possible, lightpaths and VLANs were and are being used to protect the "normal" users from disturbances caused by the transport of massive amounts of astronomical data.
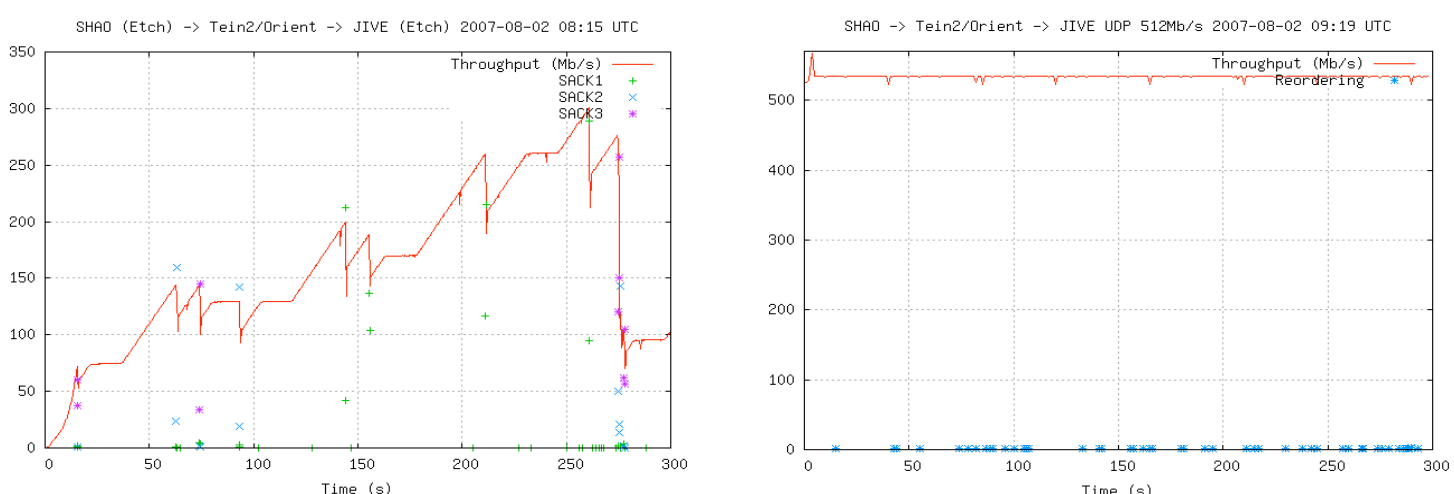

Figure 2: Throughput from Sheshan station (China) using TCP (left) and UDP (right)

One of the big advantages of doing VLBI in real time is the immediate feedback from correlator to stations. This makes it possible to identify and solve problems immediately and has meant an enormous improvement of the reliability of the network. Retrofitting the correlator, designed to process magnetic tapes in batch-mode, to handle streaming data in real-time was a major undertaking but also greatly improved the reliability of the system. This is illustrated in Figure 3, showing the data throughput to the correlator at JIVE during a typical e-VLBI science 
run. For more than 24 hours, the system did not need to be re-started, something which has never been accomplished before even with disk-recorded VLBI.

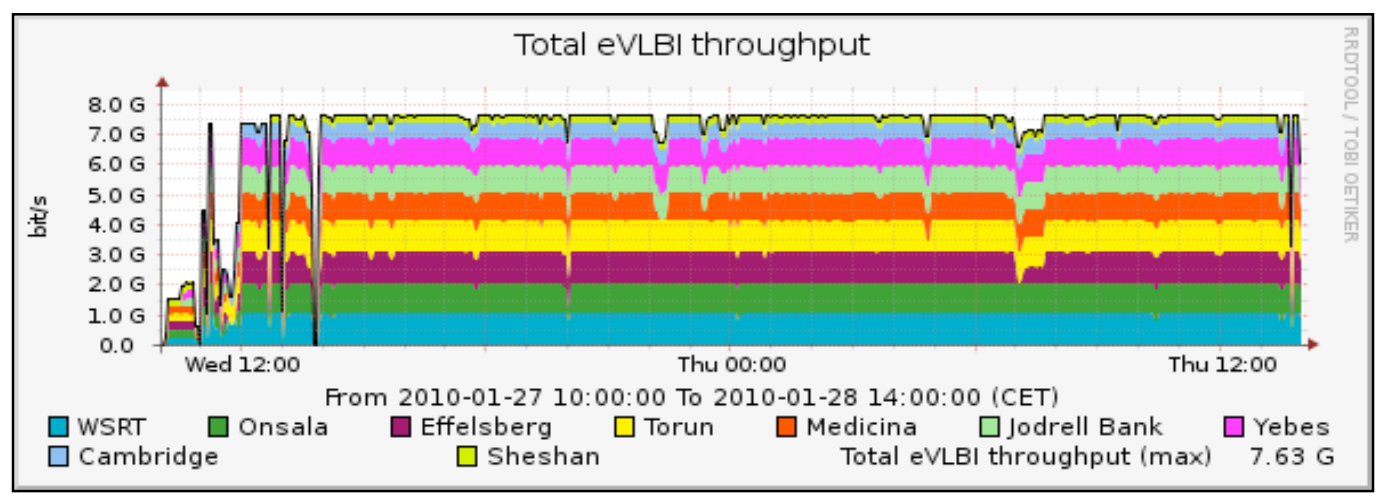

Figure 3: Data throughput into JIVE versus time during an e-VLBI run in early 2010

The question whether new science would be produced was adequately answered by the flood of publications and Astronomers' Telegrams produced since 2006. Particularly the ATs, previously practically unheard of in VLBI science, demonstrate how the new real-time capabilities have changed the type of science that is being done with the EVN. With new all-sky instruments like LOFAR coming on line, observing the transient universe will only grow in importance.

\section{The next step: NEXPReS}

In spite of the success of EXPReS, and the clear advantages e-VLBI offers with respect to robustness and rapid response, the majority of VLBI observations with the EVN still involve the recording on and shipping of magnetic media. There are a number of reasons for this.

First of all, not all telescopes have adequate connectivity (e.g. the VLBA). However, even within Europe, densely covered through its extensive network of well-funded NRENs, some stations remain practically un-connected. The Russian KVASAR network telescopes and some of the Chinese telescopes likewise have very low connectivity; moreover this situation is not expected to change for the better in the near future.

Secondly, the EVN hardware correlator is not perfect, and multiple correlator passes are still often needed. Although some of this can be remedied by the use of the EVN software correlator, developed at JIVE, the increase in number of stations and bandwidth will make realtime operations on this platform problematic.

These considerations led to the definition of the NEXPReS project. Its aim is to completely remove the distinction between traditional disk-based and e-based operations, by adding a real-time component to all VLBI observations and implementing fast and flexible 
buffering systems at both stations and correlator. Combining recorded and real-time VLBI will provide the best of two worlds, with optimal reliability, robustness and responsivity.

\subsection{NEXPReS: Novel EXplorations Pushing Robust e-VLBI Service}

The 3.5 M€ EC-funded NEXPReS (http://www.nexpres.eu/) project formally got underway on July 1st, 2010. It has 15 partners from the astronomical, networking and HPC communities, with a very high level of contributed effort. The main objectives can be summarised as follows:

1. Introduce transparent caching

2. Bring increased sensitivity, flexibility and robustness of real-time VLBI to all EVN experiments

3. Deploy a high-speed, flexible caching system

4. Allow transparent re-transmissions and/or re-correlation

5. Remove distinction between VLBI and e-VLBI operations

6. Continue collaboration with NRENs

7. Explore common technology questions with LOFAR, SKA

The project structure closely resembles that of EXPReS, continuing its successful outreach programme and interdisciplinary fora. Figure 4 illustrates the overall project setup and its division into Networking, Service and Joint Research Activities.

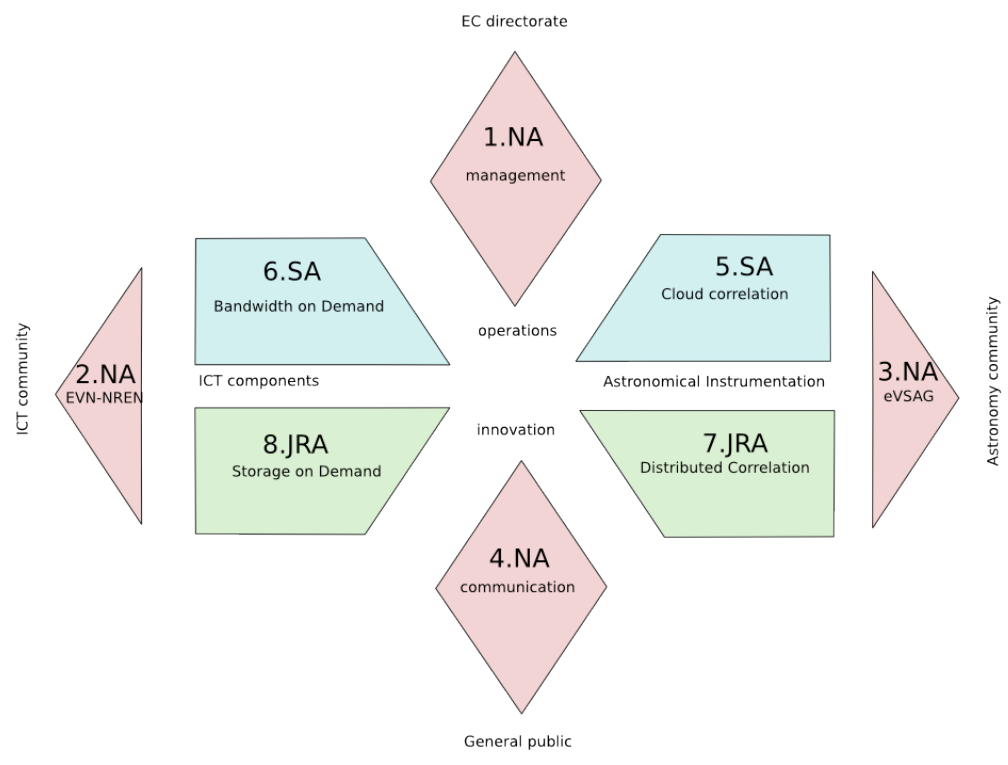

Figure 4: Schematic representation of project setup and interfaces 


\subsection{Service Activities}

Two Service Activities (SAs) are at the heart of the project.

Cloud Correlation will transform the complete EVN observational chain, from scheduling to correlation. It will enable modification of observational parameters on-the-fly, implement 4Gbps recording, transmitting and playback, with flexible buffering at the stations and correlator. Continuous quality and network monitoring will be an essential element, as will a larger amount of remote station control. A high degree of automation will be implemented in all phases of the correlation process.

High bandwidth on demand will prepare the EVN for 4 and 10Gbps real-time operations and position it to take full advantage of the emerging 100Gbps networking technology. This work package will investigate existing Bandwidth-on-Demand techniques and integrate these with e-VLBI, eventually leading to the use of international and inter-continental multi-Gbps ondemand services. It will also look into on-demand access to the very large archives that instruments such as LOFAR and Apertif will produce.

\subsection{Joint Research Activities}

The Joint Research Activities (JRAs) will push VLBI technology further, looking into distributed correlation and flexible buffering. They will focus on generic solution, capable of supporting different, not necessarily astronomical applications.

Computing in a shared infrastructure will develop generic Grid alternatives to allow realtime stream processing on a distributed correlator. This will allow additional VLBI observations with a subset of the EVN and global VLBI arrays with a minimal impact on scarce resources like disks and manpower.

High-bandwidth, high capacity networked storage will develop buffering solutions capable of the multi-Gbps simultaneous I/O streaming, needed at stations and correlator. It will also investigate hardware solutions for Long Term Archives and the allocation of resources.

\subsection{New Correlators}

The NEXPReS project will lead to an EVN that will need a next generation correlator, orders of magnitude more powerful than the current MarkIV hardware correlator. While such a new platform is under development, extensive use will be made of the EVN SFX software correlator, developed at JIVE.

\subsubsection{The SFX EVN software correlator}

The SFX software correlator was first used for the tracking of the descent of the Huygens probe onto the surface of Titan. It was further developed through the FABRIC (EXPReS) and SCARIe (NWO) projects. It is installed on a dedicated cluster at JIVE, consisting of 16 nodes with a total of 128 cores. On this hardware it will correlate 512 Mbps from 9 stations, 1024 
spectral points and 1s integration in real time. It has been used for production correlation of pulsar binning projects (Figure 5); currently multiple phase center correlation is being developed.

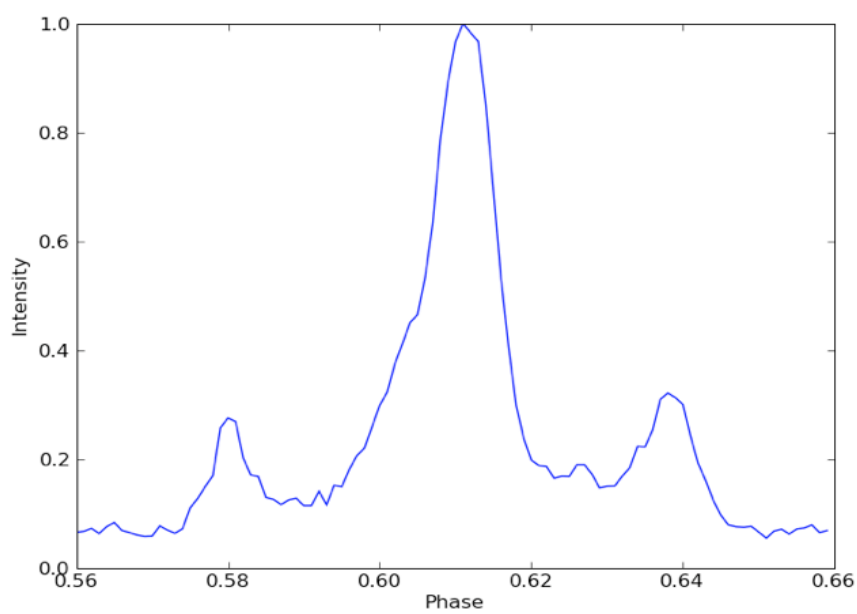

Figure 5: Pulse profile for B0329+54, obtained from 100 time bins across the pulse

\subsubsection{The UniBoard hardware correlator}

UniBoard is a Joint Research Activity in the RadioNet FP7 project. Its aim is the development of a generic, high performance, high I/O, FPGA-based computing platform, along with several astronomical applications (Figure 6). The project has nine partners, developing among others a digital receiver, a pulsar binning machine and several correlators. More information on the UniBoard can be found in [3]. This project is complemented by a joint JIVEASTRON project called the ExBox (funded by NWO) which aims at combining UniBoards into larger systems through a custom backplane.

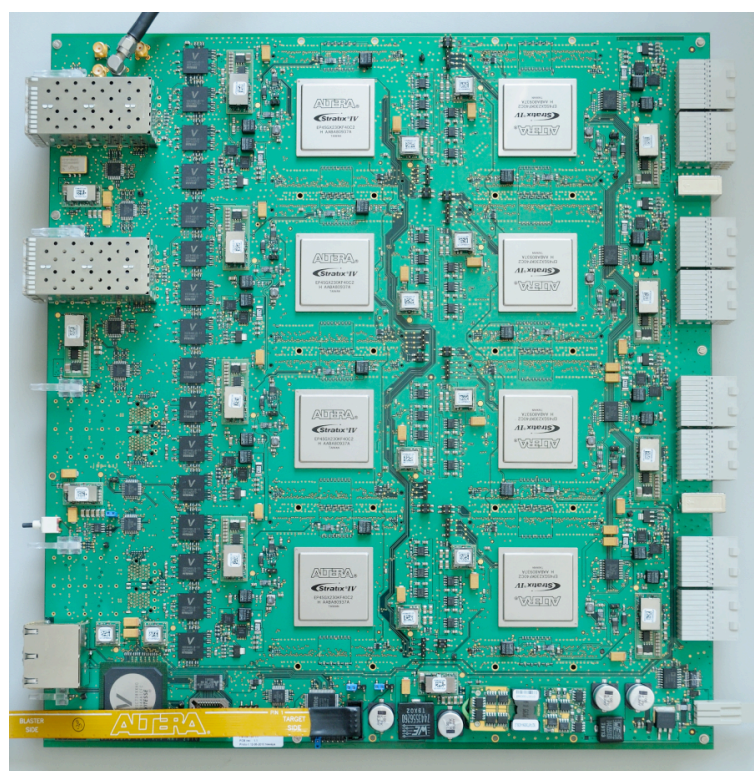


Figure 6: UniBoard prototype, delivered May 172010

As a single-board, all-station correlator, one UniBoard should be capable of processing 64 $\mathrm{MHz}$ bandwidth of 32 VLBI stations simultaneously, roughly equal to the capacity of the current MarkIV hardware correlator. With independent processing of separate frequency chunks, larger bandwidths can be handled by adding more UniBoards, while larger number of telescopes can be traded off against the bandwidth of the chunks.

\section{Conclusion}

NEXPReS will enable the transition of the EVN towards the use of real-time highbandwidth e-VLBI. Through the implementation of internationally agreed standards such as VDIF and VTP, the level of interoperability with other VLBI networks will improve. Continuous data quality and network monitoring and immediate feedback will raise the level of availibility and should lead to more frequent, more evenly spaced observing sessions. The introduction of observations with sub-sets of EVN telescopes using semi-automatically generated schedules and control mechanisms will increase the efficiency and with it the scientific yield of the EVN.

This development will help to strengthen the science case for the EVN in particular and VLBI in general during the coming years, when SKA operations get underway. Global baselines, a location which is predominantly in the Northern hemisphere and high frequencies will all remain uniquely important, certainly during the early phases of SKA. A VLBI technology roadmap will be needed, along with a strong and ambitious international collaboration. A new correlator, new receivers, new telescopes, higher frequencies and possibly stations consisting of clusters of small telescopes are innovations that will enable the EVN to stay at the forefront of radio astronomy, and remain complementary and competitive in the era of SKA.

\section{References}

[1] A. Szomoru, Recent e-EVN developments, PoS (8thEVN) 053

[2] A. Szomoru, e-EVN progress, PoS (EXPReS 09 ) 023

[3] A. Szomoru, The UniBoard, these proceedings. 